\title{
Rogue waves in the atmosphere
}

\author{
Lennart Stenflo ${ }^{1}$ and Mattias Marklund ${ }^{2}$, * \\ ${ }^{1}$ Department of Physics, Linköping University, SE-581 83 Linköping, Sweden \\ ${ }^{2}$ Department of Physics, Umeå University, SE-901 87 Umeå, Sweden
}

\begin{abstract}
The appearance of rogue waves is well known in oceanographics, optics, and cold matter systems. Here we show a possibility for the existence of atmospheric rogue waves.
\end{abstract}

Solitary waves in plasmas, and similarly in the atmosphere, have been thoroughly investigated during more than four decades [1]. Observations [2, 3] of atmospheric disturbances propagating with no appreciable change in structure are supported by theoretical descriptions where the vector nonlinearities in the atmospheric wave equations play a dominant role. Acoustic-gravity vortices in the atmosphere have thus been found [4].

Related studies of waves in the oceans have recently focused on the appearance of oceanic rogue waves (e.g. [5, 6]). Nonlinear wave studies related to the rogue wave phenomena can also be found in optics [7], superfluid-He4 [8], and optical cavities [9]. Whereas theoretical models can now fairly well describe oceanic rogue waves [10] there is, as far as we know, no corresponding theory for the atmospheric rogue waves that have been observed [11]. We shall therefore here point out a way to describe such atmospheric waves.

One should then start with the basic theory for atmospheric waves that is described in, for example, a recent review paper on nonlinear acoustic-gravity waves [12]. In contrast to most previous two-dimensional studies where the vector nonlinearities in the equations dominate, and where modon solutions thus can occur, we shall here look at the one-dimensional propagation along one of the horizontal directions (the $x$ direction). In that case only the scalar nonlinearity remains, and the slowly varying amplitude $A(x, t)$ of the normalized pressure perturbation is consequently governed by the nonlinear Schrödinger equation [13]

$$
2 i K \frac{\partial A}{\partial x}+f \frac{\partial^{2} A}{\partial \tau^{2}}-\frac{2 K^{2} n_{1}|A|^{2}}{n_{0}} A=0
$$

where $\tau=t-x / v_{0}, v_{0}$ is the linear group velocity, $K=\omega n_{0} / c$ is the wavenumber, $f=\left(K / v_{0}^{2}\right) \partial v_{0} / \partial \omega$ is the group velocity dispersion, and $n_{0}$ is the linear refractive index (here the nonlinear refractive index is given by $n_{0}+n_{1}|A|^{2}$, see Ref. [13]). We next normalize our variables, so that $x \rightarrow-K x$, $t \rightarrow K \tau /|f|^{1 / 2}$, and $A \rightarrow\left(n_{1} / n_{0}\right)^{1 / 2} A$, to obtain

$$
i \frac{\partial A}{\partial x}+\frac{1}{2} \frac{\partial^{2} A}{\partial t^{2}}+|A|^{2} A=0
$$

which, for example, has the rogue wave solution (see Fig. 1)

$$
A(x, t)=\left(4 \frac{1+2 i x}{1+4 x^{2}+4 t^{2}}-1\right) \exp (i x)
$$

It is now clearly established that the ocean rogue wave phenomenon is much more common than expected from early

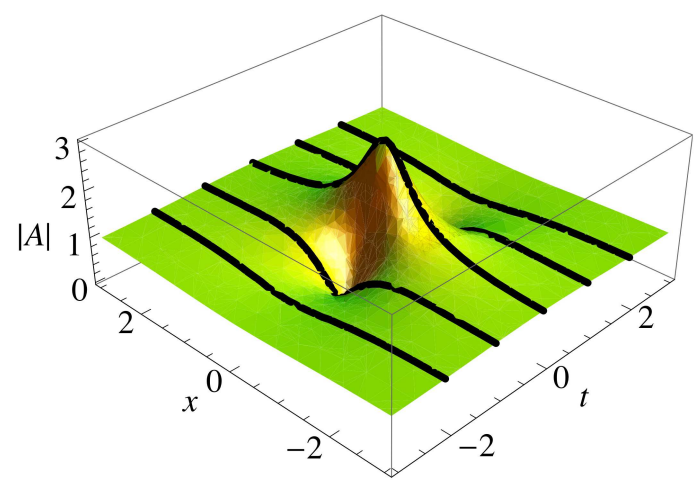

FIG. 1: The evolution of the absolute value of the solution (3).

wave modeling [14]. The description of ocean rogue waves by the nonlinear Schrödinger equation [15] bears close resemblance to the dito for atmospheric disturbances. Encounters of rogue waves on the ocean is a truly horrific experience, and it would of course be an even greater threat to meet the atmospheric counterpart while flying. Investigations of these nonlinear wave structures in the atmosphere could also be of interest for a wide community of researchers, from both basic and applied sciences.

* Electronic address: mattias.marklund@ physics.umu.se

[1] Petviashvili, V.I. and Pokhotelov, O.A., Solitary waves in plasmas and in the atmosphere (Reading: Gordon and Breach, 1992).

[2] Ramamurthy, M.K., Collins, B.P., Rauber, R.M. and Kennedy, P.C., Nature 348, 314 (1990).

[3] Widell, H.U., Bittner, M. and Hoppe, U.P., J. Atmos. Terr. Phys. 56, 1779 (1994).

[4] Stenflo, L., Z. Naturforsch. A 46, 560 (1991).

[5] Ankiewicz, A., Devine, N. and Akhmediev, N., Phys. Lett. A 373, 3997 (2009).

[6] Dysthe, K., Krogstad, H. E. and Muller, P., Annu. Rev. Fluid Mech. 40, 287 (2008).

[7] Solli, D.R., Ropers, C., Koonath, P., and Jalali, B., Nature 450, 1054 (2007).

[8] A. N. Ganshin, V. B. Efimov, G.V. Kolmakov, L. P. MezhovDeglin, and P. V. E. McClintock, Phys. Rev. Lett. 101, 065303 (2008).

[9] A. Montina, U. Bortolozzo, S. Residori, and F. T. Arecchi, Phys. Rev. Lett. 103, 173901 (2009).

[10] Marklund, M. and Stenflo, L., Physics 2, 86 (2009).

[11] Sim, B.L., and Agterberg, F.P., J. Geodynamics 41, 451 (2006); 
see their reference Gary, B.L., Rogue waves in the atmosphere (1999).

[12] Stenflo, L. and Shukla, P.K., J. Plasma Phys. 75, 841 (2009).

[13] Shvartsburg, A.B., Phys. Lett. 68 A, 281 (1978).
[14] Kharif, C., Pelinovsky, E., and Slunyaev, A., Rogue waves in the Ocean (Springer-Verlag, 2009).

[15] Zakharov, V.E., J. Appl. Mech. Tech. Phys. 2, 190 (1968). 